\title{
LA CONDICION DE L'HYSTORIOGRAPHE : ENQUÊTE SUR UNE FIGURE ET UN STATUT DANS L'EEUVE DE GEORGE CHASTELAIN
}

\author{
Estelle Doudet
}

De Boeck Supérieur | «e Moyen Age »

2006/3 Tome CXII | pages 545 à 556

ISSN 0027-2841

ISBN 2-8041-5168-9

Article disponible en ligne à l'adresse :

https://www.cairn.inforevue-le-moyen-age-2006-3-page-545.htm

Distribution électronique Cairn.info pour De Boeck Supérieur.

(C) De Boeck Supérieur. Tous droits réservés pour tous pays.

La reproduction ou représentation de cet article, notamment par photocopie, n'est autorisée que dans les limites des conditions générales d'utilisation du site ou, le cas échéant, des conditions générales de la licence souscrite par votre établissement. Toute autre reproduction ou représentation, en tout ou partie, sous quelque forme et de quelque manière que ce soit, est interdite sauf accord préalable et écrit de l'éditeur, en dehors des cas prévus par la législation en vigueur en France. Il est précisé que son stockage dans une base de données est également interdit. 


\section{La condicion de l'hystoriographe: enquête sur une figure et un statut dans l'œuvre de George Chastelain ${ }^{1}$}

Depuis l'assertion d'A. Molinier dans Les sources de l'histoire de France en 1904, il est devenu commun de répéter qu' « à la fin du Moyen Âge, l'histoire s'est faite bourguignonne ${ }^{2}$ ». Des Rhétoriqueurs aux mémorialistes, l'écriture historiographique en Bourgogne a elle-même une histoire, placée sous le double signe de la varietas et de la copia. Variété et richesse d'un genre qui sont liées à l'éclosion d'une culture propre à cet espace septentrional, soutenue par une volonté politique originale. La puissance du duché Valois, au cours de ce $X V^{\mathrm{e}}$ siècle où il domine les affaires européennes, s'exprime en effet non seulement par les armes et la diplomatie, mais par l'affirmation d'une autonomie culturelle, dont l'écriture historiographique est l'un des plus efficaces moyens. Qu'est-ce qu' un historiographe officiel bourguignon, lorsque cette fonction est confiée pour la première fois en 1455 à George Chastelain ? Qu'est devenu ce statut vingt ans plus tard, en 1473, lorsque le même auteur reçoit de Charles le Téméraire un nouveau titre :l'indiciaire ?Ce terme, créateur d'une sorte de conscience dynastique chez les successeurs de Chastelain et qui sera le moteur d'une réflexion chez Jean Molinet et Jean Lemaire de Belges, ne couronne que fort tardivement la carrière du premier Rhétoriqueur. Entérinant la réussite d'une entreprise autant historiographique que rhétorique, le statut d'indiciaire puise ses sources dans ce que Chastelain nomme quant à lui la condicion del'hystoriographe. D'un titre à l'autre, d'une génération à la suivante, au cours de l'œuvre de Chastelain se dessine une recherche sur l'écriture de l'histoire à laquelle la définition problématique de ce qu'est un « Rhétoriqueur » semble profondément liée.

1. Pour connaître l'expression complète des titres abrégés tout au long de ce travail, le lecteur voudra bien se reporter à la bibliographie thématique du sujet traité, située à la fin de la section renfermant les présents actes des rencontres de Dunkerque consacrées aux Littérature et culture historiques à la cour de Bourgogne.

2. Molinier, Sources de l'histoire de France, t. 4, p. 186. 
$\mathrm{Si}$ « l'histoire s'est faite bourguignonne » à l'automne du Moyen Âge, il est aussi évident qu'elle est née française et que c'est à partir et contre le modèle des chroniqueurs dyonisiens que les ducs de Bourgogne ont imaginé la création d'une historiographie officielle de leurs États. Bien que la tradition des Grandes Chroniques soit plus ancienne, la politique vernaculaire de Charles $\mathrm{V}$, puis les troubles civils du début du $\mathrm{XV}^{\mathrm{e}}$ siècle donnent à leur rédaction en langue française un nouvel essor, dont témoigne l'œuvre de Michel Pintoin, le Religieux de Saint-Denis ${ }^{3}$. La rénovation formelle des Grandes Chroniques va de pair avec une conscience réaffirmée, de la part des monarques, de l'importance politique d'une historiographie contemporaine. On sait que Charles VII, lors de sa Joyeuse Entrée à Paris en 1437, a pour premier geste la nomination de Jean Chartier comme chroniqueur du royaume. Celui-ci, dans le prologue de sa Chronique, ne manque pas de souligner l'importance symbolique de ce geste de souveraineté retrouvée :

Cy commence la cronicque du temps de tres chrestien roy Charles septiesme de cenom, roy de France, faicte et compilée par moy, frere Jehan Charretier, religieux et chantre de l'eglise Monseigneur saint Denis, cronicqueur dudit royaulme, àce commis, ordonné et député de par le roy mon souverain seigneur, combien que à icelle faire suys et me reputte moindre de tous et entre les aultres plus indignes ${ }^{4}$.

Le terme de croniqueur sur lequel Jean Chartier insiste est l'objet à cette époque d'une spécialisation sémantique, comme l'a montré J. Devaux ${ }^{5}$. Aucun écrivain ne peut l'obtenir sans mandat officiel. Le prologue de l'historien breton Alain Bouchart vers 1500 est encore formel à cet égard : la cronique est un livre [...] composé par celuy qui à ce faire a esté commis, car il n'est permis à personne composer cronique s'il n'y a esté ordonné et deputé.

La chronique royale de Charles VII s'affirme donc, malgré les topoï de modestie de Jean Chartier, comme une écriture dont la puissance est à la fois mémorielle et contemporaine : raconter le passé, magnifier le présent.

3. M. Pintoin (Religieux de SAint-Denis), Chronique contenant le règne de Charles VI, de 1380 à 1422, éd. et trad. L. Bellaguet et B. Guenée, 6 t. en 3 vol., Paris, 1994. Cf. B. Guenée, L'Opinion Publique à la fin du Moyen Âge d'après la Chronique de Charles VI du Religieux de Saint-Denis, Paris, 2002.

4. J. Chartier, Chronique de Charles VII, éd. A. Vallet de Viriville, t. 1, Paris, 1858, p. 25.

5. Devaux, Jean Molinet, indiciaire bourguignon, p. 25-46.

6. A. Bouchart, Grandes croniques de Bretaigne, éd. M.L. Auger et G. Jeanneau, t. 1, Paris, 1986, p. 77. Furetière, à la fin du XVII e siècle, substitue son sens à celui d'historiographe. «Chroniqueur» prend une connotation neutre, alors que son doublet se spécialise. «Historiographe : celuy qui a escrit ou qui escrit l'histoire. On le dit plus particulièrement de ceux qui ont une commision, un brevet particulier pour escrire l'histoire de leur temps ». A. Furetière, Le Dictionnaire universel, réd., t. 2, Paris, 1984, non paginé. 
Il n'est guère étonnant qu'une vingtaine d'années plus tard, la volonté $\mathrm{d}^{\prime}$ une concurrence culturelle face au royaume conduise Philippe le Bon vers la création d'une historiographie propre à la Bourgogne. Cette décision n'intervient pas au hasard. En 1454, le duc est conscient d'une forme d'apogée de son principat. La paix règne dans ses États dont l'ampleur et la richesse sont uniques. Le Banquet du Faisan, organisé à Lille, confirme aux yeux de l'Europe son statut de rempart de l'Occident contre les menaces orientales. Le souci dynastique de Philippe se manifeste cette même année par les négociations matrimoniales qui aboutissent au mariage de son fils Charolais et de l'héritière des Bourbons, nouveau lien diplomatique avec la France. En juin 1455, comme une sorte de couronnement de cette politique de prestige et de pérennité, Philippe le Bon assigne à George Chastelain la tâche d'inscrire les faits de son maître dans la mémoire des hommes. La notion de « mise en forme », clef de la future œuvre historiographique du Rhétoriqueur, apparaît dans la définition officielle de sa mission par les instances ducales :

Par consideration de ce qu'il est tenu de mettre par escript choses nouvelles et moralles, en quoy il [est] expert et congnoissant, aussi mettre en fourme par maniere de cronicque fais notables dignes de memoire advenus par chi devant et qui adviennent et puellent souvente fois advenir ${ }^{7}$.

Il est remarquable que Chastelain, lorsqu'il parle de son activité d'historien, n'use pas du titre prestigieux de croniqueur, pourtant impliqué par les documents qui le nomment à ce poste. Il se peint toujours comme un hystoriographe, employant volontairement le terme le plus neutre. Deux premières raisons peuvent être convoquées pour expliquer ce choix. L'auteur revendique sans doute par ce biais une position humble, topos qui n'est pas propre au discours du Bourguignon. D'autre part, Chastelain, en préférant se dire hystoriographe, use d'un terme qui lui permet de neutraliser les menaces de partialité qui pèsent sur son œuvre. Dans Les Exposicions sur Verité mal prise, adressées au début de 1461 à un public français assez réticent, l'écrivain se désigne dès son prologue comme le moindre des hystoriographes franchois, ce qui est une habile manière de s'intégrer à la tradition française des hautes esmerveillables chroniques, tout en masquant le fait que, chroniqueur officiel des Valois bourguignons, il fait œuvre de propagande en faveur de son duc. Autre raison peut-être, qu'il faut puiser dans le prologue élaboré qu'il donne à sa Chronique au moment où il commence sa rédaction. L'écrivain évoque la lignée d'historiens dont il s'inspire et indique ses buts. Il

7. Lille, Archives départementales du Nord (= A.D.N.), Collection des Acquis pour les Comptes du Grand Sceau, B. 9880, fol. 29. Chastelain reprend cette formulation en 1473 : J'ay vacquié et entendu a mectre par escript en fourme de cronike fais notables et digne de memoire a l'ordonnance de mon devant dit tresredouté seigneur [...] (A.D.N., B. $\left.2050, n^{\circ} 63694\right)$. 
entend exprimer la gloire du présent, ressusciter le passé pour édifier (aux deux sens du terme) les lecteurs à venir :

Je doncques, George Chastellain [...] ay prins et recueilly devers moy les escris des historiographes nouveaux de mon temps, avec ce que de mon costé y ay veu et congnu, et sur toutes les choses escriptes à Saint-Denys et aussy par autres de ce temps, ay fait concordance et espluchemens de vérité $[\ldots]^{8}$.

Sources prestigieuses mais éparses, peut-être fautives, les œuvres dyonisiennes doivent être soumises au travail de concordance, c'est-à-dire de totalisation et d'équilibrage de l'information, en vue de transmettre la senefiance d'un événement que le témoignage personnel de Chastelain assure d'autre part. L'humble hystoriographe, metteur en forme de l'histoire, insiste sur le fait qu'iln'est ni clerc ni moine, comme le sont ses homologues de SaintDenis :

Né en l'imperialle conté d'Alost en Flandres, extrait de la maison de Gavre et Mammynes, sobrement instruit ès lettres, nourry en fleur de jonesse ès armes et en la hantise des cours royales et nobles hommes, souverainement des Franchois, enaigri durement ès armes et exercité soubz longues annuyeuses contraires fortunes $[\ldots]^{9}$.

Ces quelques lignes à la première personne dessinent le portrait d'un laïc, familier des milieux curiaux, aristocrate flamand cultivé, connaisseur des armes plus que des lettres ; un conseiller de Philippe le Bon, connu de la cour de France et que sa carrière militaire autant que diplomatique désigne comme relateur de la gloire chevaleresque de son prince. George l'Adventurier est tout entier dans cette image. Pourtant, depuis les études de Gr. Small ${ }^{10}$, on sait que la généalogie aristocratique que se donne ici Chastelain est un leurre. Messire George est en fait d'origine bourgeoise et flamande. Le coup de force de l'historiographe est de se fabriquer une identité mensongère pour défendre la véracité de son écriture. S'il faut voir dans l'affirmation d'une noblesse d'emprunt une manœuvre de rapprochement complice avec le commanditaire ducal et le public de la cour, on peut également y lire la volonté de s'éloigner du modèle clérical traditionnellement lié au statut de chroniqueur. Dans l'ensemble de son œuvre, en effet, Chastelain refuse de s'assimiler à la représentation habituelle du clerc, dont l'influence sera encore prégnante chez Jean Molinet ${ }^{11}$. Le statut décalé des clercs-chroniqueurs face à une

8. G. Chastelain, OEuvres, éd. Kervyn de Lettenhove, t. 1, p. 11-12, texte corrigé sur le manuscrit source d'Arras (ArRAs, Bibliothèque Municipale, ms. 516, fol. 8 v $^{\circ}$ ).

9. Ibid., t. 1, p. 11, corrigé sur le même manuscrit (fol. 8).

10. SMALL, George Chastelain and the Shaping of Valois Burgundy, p. 9-50.

11. Jean Molinet revient au portrait clérical, caractérisé par la pauvreté, la laideur topique et la position décalée en société. Son statut social réel est en effet plus net que celui de Chastelain puisqu'il est chanoine de Valenciennes. Il est enclin à se figurer boiteux ou louche, porteur d'un regard oblique caractéristique de celui du clerc, tout 
société active cède chez Chastelain à une figuration de soi certes fictionnelle, mais qui a pour but d'inventer un statut original, celui d'un historiographe laïque et engagé, conservant, par sa maîtrise de l'écriture et par la mission que lui a confiée son maître, l'auctoritas d'une voix officielle.

Le topos de modestie exprimé par le terme hystoriographe cache donc une ambition novatrice. Si les sources auxquelles Chastelain puise sont désignées par lui comme croniques, ses propres écrits ne sont décrits que comme simple relation $^{12}$, modeste peinture ${ }^{13}$ ou memoire ${ }^{14}$. Les digressions de la Chronique tracent de l'auteur un double auto-portrait : il se définit comme un orateur, prononcheur ${ }^{15}$ ou reciteur de vérité et comme un preud'homme greffyer ${ }^{16}$, dont le stylet grave avec exactitude l'événement. À l'humilité insistante et suspecte de l'hystoriographe répond souvent un autre terme, la legende que ses textes rêvent d'être. Le sens ancien d'hagiographie attaché à ce terme s'étend chez Chastelain jusqu'à qualifier le caractère sacré à ses yeux d'une écriture qu'il conçoit comme historique et encomiastique à la fois. Legenda : impératif de lecture, miroir exemplaire offert aux hommes à travers le temps, rêve d'une nouvelle historiographie, prise dans les tensions de la véracité impartiale qui la fonde et de la glorification du présent vers laquelle elle tend.

Si l'historiographe chez Chastelain se défend d'être chroniqueur, qu'estil ? Avant toute chose, et selon la mission donnée par Philippe le Bon, un « metteur en forme » en charge de la concordance qui fait de la confusion du réel un texte au sens étymologique, tissage de faits, de témoignages, d'interprétations. De même que Dieu esquisse des hiérarchies entre les hommes et entre les événements, selon Chastelain, l'historiographe doit non tout dire, mais travailler son texte pour que s'y exprime une verité espluchée, assumée comme un choix.

Cette réflexion, qui fonde ce que Chastelain nomme la condicion de l'hystoriographe, imprime à l'œuvre un rythme particulier, souligné par l'ensemble de la critique comme " digressif ${ }^{17}$ ». La Chronique de Chastelain,

en conservant les acquis de l'orateur. Cf. J. Cerquiglini-Toulet, L'écriture louche. La voie oblique des Grands Rhétoriqueurs, Les Grands Rhétoriqueurs. Actes du Ve colloque International sur le Moyen Français. Milan 6-8 mai 1985, t. 1, Milan, 1985, p. 21-31.

12. G. Chastelain, CEuvres, t. 6, p. 163 (Lion Bendé).

13. Ibid., t. 6, p. 457 (Mort de Charles VII) : Lettre et nouvelle peinture.

14. Ibid., t. 7, p. 201 (Recollection des merveilleuses advenues) : Ceste brefve memoire de tant de divers dits.

15. La description d'une gloire historique récitée par une voix vive, proferée avec sacralité, s'exprime dans ce terme fréquent, revêtu lors de ses occurrences d'une grande importance : prononcheur de verité (ibid., t. 4, p. 95), profereur de verité sainte (G. Chastelain, Exposicions sur Verité mal prise, éd. Delclos, p. 185).

16. ID., CEuvres, t. 4, p. 95.

17. L'importance des digressions chez George Chastelain a été soulignée dès les introductions de son éditeur J. Kervyn de Lettenhove et les premiers travaux sur la 
que l'on juge cela irritant ou remarquable, est unanimement considérée comme un récit sans cesse interrompu par des réflexions menées à la première personne sur le sens de l'événement relaté. Est-ce l'hypertrophie d'une tendance digressive propre à l'époque ou la conséquence d'un statut de parole particulier, celui d'historiographe selon Chastelain ? Choisissant cette seconde hypothèse, nous proposons de voir dans l'écriture historique de George, non un texte digressif, mais le déploiement simultané de deux types de discours : une parole linéaire, rapportant des contingences et s'exprimant dans la succession des chapitres ; un langage répétitif, celui de la «digression", qui est en fait un geste de généralisation morale qui transforme le hasard en nécessité et la politique en littérature. Loin d'être une réaction secondaire, un détour ajouté à la narration, la « digression » propose au texte et à son lecteur l'horizon du vrai sens ${ }^{18}$.

On peut ainsi étudier l'intéressant travail de mise en forme opérée par George sur le texte d'Enguerrand de Monstrelet, dont la Chronique forme la source des deux premiers livres de Chastelain. Chez Monstrelet, les premières lignes de chaque chapitre s'ouvrent par des informations temporelles peu travaillées et essentiellement factuelles : en ce temps [...], en ce jour de [...]. Dans plus des deux tiers des occurrences chez George, les incipit sont au contraire le lieu d'une mise en scène de la voix narrative. Les deux écrivains rapportent ainsi l'attaque de Corbie en 1431 par les troupes de Charles VII :

\begin{abstract}
En ce mesme temps, se assemblerent plusieurs des capitaines du roy Charles, est assavoir le seigneur de Longueval, Anthoine de Chabennes, Blanchefort, Alain Geron et plusieurs autres. Si chevaucherent tous ensamble avec leurs gens vers la ville de Corbie, laquelle ils cuiderent prendre par soudain assault ${ }^{19}$.

Ja soit ce que longuement me suis tu des Françoys, comme s'ils dormissent et laissassent en paix ceux de leur parti contraire, Bourgongnons et Angles, toutevoyes, parce que je les treuve veillans tousjours et soigneux en labeur ça et la pour acquerre et gagner ou los ou profit, ou tous deux quant il y eschiet, il me convient escrire maintenant comment le seigneur de Longueval, qui s'estoit tourné Françoys, ensamble messire Jacques de Chabannes, Blanchefort, Alain Geron et pluiseurs autres tenant les frontieres s'estoient assamblés de pluiseurs garnisons et mis es champs à intention de venir à Corbie ${ }^{20}$.
\end{abstract}

L'historiographe George impose la première personne et justifie la réinsertion des personnages dans le récit, ce que le texte plus linéaire de Monstrelet

Chronique, comme ceux de B. Renard (B. RENARD, Quelques observations à propos de quatorze chapitres inédits de Georges Chastellain, Trésor National, 1842, p. 91-156). G. Pérouse parle d'écriture «psychologique » et « généraliste » (Pérouse, Georges Chastellain, p. 41).

18. Nous nous permettons de renvoyer sur ce point à notre ouvrage : DOUDET, Poétique de George Chastelain, p. 377-393.

19. E. De Monstrelet, Chronique, éd. DouËT-D'ArCQ , t. 1, p. 439.

20. G. Chastelain, CEuvres, t. 2, p. 201. 
ne faisait pas. Le Rhétoriqueur ajoute un détail signifiant. Dès que la focalisation ne se porte pas sur un personnage, celui-ci s'assoupit (comme s'ils dormissent), puis se réveille et réintègre l'histoire-récit. Metteur en scène, metteur en forme, l'historien est le re-créateur d'une réalité, qui n'est plus celle du monde, mais celle du livre. La conception de la narration dans la Chronique est assez semblable à la pratique théâtrale contemporaine de George : tout est potentiellement visible, mais seule la lumière proposée par le discours de l'historiographe permet aux personnages de quitter l'arrièrescène pour vivre leur aventure devant les spectateurs.

À cela s'ajoute une reconfiguration générale de la source. La relation du siège de Compiègne, où Jeanne d'Arc est faite prisonnière et où Bourguignons et Armagnacs s'affrontent en un long combat, illustre ce fonctionnement de l'écriture. Le texte de Chastelain glisse du récit événementiel offert par Monstrelet à une narration personnalisée, pour aboutir au commentaire :

Ouquel temps aussi, en faisant lesdictes mines et approches, en y eut pluiseurs des assegeans mors et navrés. Desquels mors furent les principaux, messire Jehan de Belles, chevalier, Alard d'Escassines, Thiebault de Caitignies et pluiseurs autres, tant de la partie de Bourgogne, comme des Anglois ${ }^{21}$.

Sy fut fait ledit fossé à coup, et fut de grant fruit aux approchans et leur fit de service beaucoup, car mortellement dru venoit le trait de dedens sur eux tant de canons comme de couleuvrines, dont il en y avoit de bons ouvriers avecques eux, par especial un cordelier natif et vestu à Valenciennes, nommé Noiroufle [... $]^{22}$.

Passant sous silence les morts et les blessés bourguignons et inversant ainsi l'analyse de la situation militaire, de la défaite en possible victoire, Chastelain privilégie la description des assiégés français, résumée en un portrait de Noiroufle. Cette focalisation se fait sous le signe de la merveille, couleur hyperbolique caractéristique du passage du récit au discours dans la Chronique:

[...] un cordelier natif et vestu à Valenciennes, nommé Noiroufle, un haut grant homme noir, atout un laid murtrier visage et une felle veue et un grant long nez et portoit rude grosse faconde et semblant espoventable entre tous les autres d'eglise et de religion (de tous ceux que je vis oncques, le moins apparant homme d'eglise).

L'attention que l'historien accorde à ce personnage est justifiée par une certaine familiarité : une même origine géographique (Valenciennes où Chastelain vit) ; un homme connu personnellement par le narrateur. Les adjectifs négatifs, les topoï du vilain et du criminel et jusqu'au nom presque programmatique de Noiroufle : tout contribue à tracer un portrait inquiétant, sous le signe de la cruauté, de l'hypocrisie religieuse et de l'opacité (le moins

21. E. De Monstrelet, Chronique, t. 1, p. 391.

22. G. Chastelain, OEuvres, t. 2, p. 53. 
apparant homme d'eglise). Quelle est la raison de cet ajout? En Noiroufle, Chastelain résume les défauts de l'ennemi : prêtre oublieux de Dieu, assoiffé de sang, l'homme tisse un lien assez net avec Jeanne d'Arc, deux chapitres plus haut. Noiroufle dans l'ombre de la Pucelle jette une obscurité fâcheuse sur les Armagnacs. Mais le portrait ne s'arrête pas là, car l'avenir de Noiroufle rejoint le présent biographique du narrateur :

Maint an vesqui apres toutevoyes [... ] et vint jusques à estre de la retenue du roy
et de son hostel et bien privé de luy. Souvent disoit messe devant luy, la ou je l'ay
vu et bien congnu et esté en maintes devises par diverses fois bien privées. Dont
quant je le vis chanter messe et me recordoye de la multitude des murtres horribles
qu'il avoit fait en commune renommée et que, avecque ce je regardoye la forme et
la physionomie de luy, qui estoit de mesme aux faits qu'on luy attribuoit, souvent
me suis espovanté en moy mesme de la hideur et m'en sont les cheveux dressés
contre-mont, disant à par moy comment gens de telle grace, et encore en estat qui
contredit entierement à leurs faits, peuvent avoir reception en court [...].

Le portrait de Noiroufle offre deux points d'ancrage pour l'architecture symbolique de l'œuvre. Politiquement, ce prêtre-soldat incarne la figure de l'adversaire, rejaillissant sur celle de Jeanne d'Arc et postérieurement sur Charles VII, souverain aveugle qui le reçoit à sa cour. D'un point de vue narratif, cet arrêt permet le glissement de l'événementiel narré au commentaire à la première personne. Il est enfin l'occasion de voir resurgir, au détour d'un chapitre, les interrogations fondamentales de l'esthétique de George : la représentation est-elle possible dans un monde où le rapport des paroles et des actes est trouble, où le prince est aveugle et Fortune souveraine? La complexité et le degré d'achèvement d'une telle construction, éloignée de l'écriture habituelle de la digression, permet de comprendre, nous semble-til, pourquoi Jean Molinet, héritier de Chastelain, évitera de surenchérir sur les mêmes techniques narratives et choisira de fonder une autre rhétorique, modelant de l'intérieur la parole et travaillant l'ornement.

L'histoire selon George implique la participation active de l'écrivain au texte et sa position d'interprète face aux faits. Ces principes ne cachent pas pourtant les ambiguités qui traversent une historiographie commandée. Lorsqu'il accède à la position de chroniqueur officiel, position dont il va faire évoluer les présupposés vers le nouveau statut d'hystoriographe, George se voit contraint de répondre à une double exigence : morale et historique - il faut laisser à la postérité une juste peinture de son maître ; encomiastique il faut fixer les traits d'une gloire rayonnante in saeculi saeculorum. Le discours de l'historiographe bourguignon n'est pas seulement mise en forme de la réalité ou de sources antérieures. Répondant de façon précise aux exigences de la nomination de 1455, il est un travail « d'expert » en rhétorique, d'orateur, qui doit, au sein de l'écriture de l'événement, faire resurgir et mêler les styles aristotéliciens : le délibératif ou la morale de l'action, l'épidictique ou l'équilibre de la louange, le judiciaire ou la défense de la vérité. 
Il est impossible à Chastelain de dissocier la morale de son écriture historiographique, puisque cette dernière repose sur une constante argumentation éthique. Le système de l'éloge et du blâme ne peut se comprendre pour le Rhétoriqueur hors des deux autres genres rhétoriques. Mais il ne peut leur être subordonné puisque le dessein profond de l'œuvre est l'établissement hic et nunc de la gloire pour le commanditaire. Comment résoudre ce problème ? Chastelain choisit de bouleverser la hiérarchie des genres rhétoriques. L'éloge ne sera pas une simple possibilité d'écriture parmi d'autres, mais une structuration générale, le but ultime de la Chronique. Mais pour que ce travail ait lieu avec efficacité et discrétion, il convient de le situer dans un genre médiéval traditionnellement peu soumis au système oratoire, les chroniques. Le narratif historiographique se transforme, sous la plume de Chastelain, en discours à but encomiastique. Le chroniqueur se révèle Rhétoriqueur.

L'hystoriographe selon Chastelain est-il le modèle de ces écrivains biens excellents que dénonceront, quelques décennies plus tard, Montaigne, et après lui de nombreux lecteurs modernes ? Dans un texte célèbre, l'auteur des Essais distingue les historiens simples, comme Froissart, qui montrent la matière de l'histoire nue, et les biens excellents qui ont la suffisance de choisir ce qui est digne d'estre sçeu, et peuvent trier de deux rapports celui qui est le plus vraisemblable ${ }^{23}$.

Le critère de ce que l'on pourrait nommer, à partir de Montaigne, «le vraisemblable encomiastique » est en effet au cœur du problème. Il structure le remarquable texte que Chastelain consacre à la vraye condicion de $l^{\prime}$ hystoriographe $e^{24}$. Répondant par avance à une critique qu'il sent possible envers ses descriptions enthousiastes de la magnificence bourguignonne lors de la Joyeuse Entrée de Louis XI à Paris en 1461, George fait intervenir dans une digression la voix fictive de lecteurs de mauvaise foi, qui remettent en cause les principes de son écriture :

Me semble povreté ceux qui disent: "Tel et tel escript en faveur d'un tel et le collaude et blasonne ». Mais sous correction de ceux qui ainsi parlent, ou ils sont mal informés des besongnes et les ont mal enquis, ou ils ignorent la condicion de l'hystoriographe [...].

Qu'est-ce donc qu'un historiographe ? Avant tout un Rhétoriqueur, c'est-àdire un dispensateur de l'éloge et du blâme. Son choix des couleurs encomiastiques n'est pas aveugle. L'historiographe est un vray delateur des choses $^{25}$, proportionnant la richesse de sa rhétorique à la profondeur signifiante de la réalité, pour faire du flux temporel une legende creable tout en

23. M. De Montaigne, Essais, éd. P. Villey et V.L. Saulnier, t. 2, Paris, 1978, p. 416417 (liv. 2, chap. 10).

24. G. Chastelain, OEuvres, t. 4, p. 94-96.

25. Ibid., t. 4 , p. 95. 
conservant son historicité. Cette protestation d'équivalence entrel'ornement $\mathrm{du}$ discours et la gloire obtenue par l'acte historique, familière au lecteur de Chastelain, coupe court aux reproches. Elle pose pourtant de nombreux problèmes. L'historiographe, souligne George, doit juger la réalité selon le critère du vraisemblable, soutenu par l'opinion publique et sa propre raison : il faut attester la gloire lorsque par jugement universel il a esté trouvé tel. Ceux qui mettent en doute cette évidence se condamnent à la contradiction :

Responde icy qui voudra et dise que non, pour savoir si sa propre raison bien examinée ne le desjugera après et l'accusera de son tort.

Mais qui forge l'opinion, qui influence la raison bien examinée du lecteur, sinon ce greffyer qui prétend lui obéir et qui concentre en ses mains les couleurs de l'éloge et du blâme ? Au détour de cette réflexion, celui qui en equité chemine laisse échapper un autre dangereux détail. La verité droituriere qui contraint le preud'homme d'avoir l'ail à ce qui vray est trace le devoir de l'historiographe. Celui-ci est responsable de son écrit non uniquement face au prince commanditaire auquel il n'en réfère que de façon contingente, mais surtout face au Juge universel :

Donc, le prononcheur qui la publie fait service à Dieu par magnifier ses creatures, esquelles il a ouvré de ses vertus ${ }^{26}$.

Mais quel est alors le statut du blâme? Indice d'une faille dans cette harmonie rêvée entre l'histoire réelle, la beauté du texte historique et la gloire de Dieu, le vitupere, autre face de la rhétorique, est passé sous silence :

Si escripvains labeurent pour relenquir au monde la memoire des choses passées, et que les mauvaises se peuvent taire par courtoisie, et les bonnes divulger par merite $[\ldots]$.

Tel est bien l'écueil majeur du vraisemblable encomiastique : l'attention passionnée à la louange entraîne souvent, sur des faits moins glorieux que l'on pourrait reprocher aux Bourguignons, un silence dont la « courtoisie » n'efface pas le mensonge. Si n'est vraisemblable que ce qui est objet d'éloge, comment la louange pourrait-elle sans le blâme être elle-même vraisemblable ? Pour éviter l'écroulement de son édifice rhétorique et la mise en danger du statut d'historiographe, Chastelain propose une solution : il s'agit de façonner, au sein de la Chronique, un pôle stable du vitupere, qui laissera d'autant mieux rayonner la louange. George désigne donc, sous les traits de l'Autre (Infidèles, Anglais, Armagnacs assassins), un mal stabilisé et reconnaissable, qui laisse libre cours à la gloire bourguignonne ${ }^{27}$. Mais il arrive que les vicissitudes du réel, les ambiguiités diplomatiques et les manœuvres des princes mettent en danger le système. La Chronique semble alors, comme le

26. Ibid., t. 4, p. 96 .

27. Sur ces constructions, cf. DoudET, De l'allié à l'ennemi. 
château de Fortune, un édifice magnifique, côtoyant dangereusement le précipice de sa propre destruction ${ }^{28}$.

En 1455, Philippe le Bon confie à Chastelain un poste de chroniqueur visant à concurrencer l'entreprise des Grandes Chroniques de France. De façon subtile, George accomplit le dessein de son duc, mais en déplaçant le défi. Il choisit de ne pas imiter l'historiographie française en dupliquant ses acquis littéraires et statutaires, mais d'inventer plutôt une autre figure del'écrivain : l'historiographe Rhétoriqueur, dont l'originalité fera écho à la spécificité de la culture bourguignonne. C'est désormais du sein de l'historique que doit surgir la gloire éternelle, comme c'est la narration du contemporain qui justifie et soutient le passage aux couleurs oratoires du discours. Le rôle de l'historiographe ainsi compris n'est pas de rendre l'existentiel, mais de dévoiler, dans $l^{\prime}$ histoire et à partir d'elle seule, $l^{\prime}$ essentiel $^{29}$. Les innovations qui fondent la réussite de l'œuvre géorgine portent également en germe les possibilités de sa défaite : manipulation dangereuse de la figure de l'écrivain qui prétend être ce qu'il n'est pas, périls d'un double rythme de l'écriture entre faits rapportés et interprétation, menaces de déséquilibre dans l'édifice fragile des genres oratoires. Au fil de ses pages, la Chronique de Chastelain relate la grandeur et les misères d'un État exceptionnel en mettant à l'épreuve, comme un curieux écho, sa propre légitimité historique. Au lecteur à venir appartiennent la compréhension et la survie de l'entreprise :

O vous, humains cœurs des Franchois qui, par successives generations de pere en fils, en temps advenir trouverez mes escripts, lorsque la main pourrie en terre reposera soubs divine mercy, si faim peut vous prendre de visiter mes euvres, et que loisir vous puisse traireà l'advertance d'icelles, veuilliez doncques noter tant seulement le son des paroles, mais les causes et racines qui m'ont mu à les former telles ${ }^{30}$.

Université Charles-de-Gaulle - Lille 3

Estelle DoudET

28. Ces difficultés, présentes tout au long de la Chronique, s'accroissent avec l'avènement de Louis XI en 1461. Les stratégies rhétoriques de Chastelain ont alors de plus en plus de mal à faire face à une réalité troublée qui met en danger les relations franco-bourguignonnes. L'échec de la Chronique, laissée inachevée à la mort de son auteur en 1475, préfigure l'écroulement de l'État bourguignon lui-même deux ans plus tard. Cf. Doudet, Poétique de George Chastelain, p. 300-314.

29. J. Blanchard souligne cette différence : « La réalité [chez les Rhétoriqueurs], quoique dominée, n'est pas véritablement rendue dans son aspect existentiel » (Blanchard, Commynes l'Européen, p. 396). Il nous semble qu'il n'y a pas lieu d'opposer péjorativement, selon un topos critique encore répandu, la liberté de Commynes à la contrainte des Rhétoriqueurs, mais qu'il s'agit plutôt de comprendre les raisons idéologiques et rhétoriques de leurs divergences.

30. G. Chastelain, OEuvres, t. 4, p. 14-15. 


\section{DROITS ÉLECTRONIQUES RÉSERVÉS SUR IMAGE(S)}

Le duc Charles le Téméraire écoute le discours que lui adressent les figures allégoriques de Clair Entendement et Congnoissance de toy mesme. George Chastelain, Advertissement au duc Charles, Maître de Marguerite d'York, après 1467, B.N.F., ms. fr. 1217, fol. $1 \mathrm{r}^{\circ}$ (@ B.N.F.) 